\author{
Vladimir Rouvinski* \\ Universidad Icesi (Cali, Colombia) \\ vrouvinski@icesi.edu.co
}

\title{
Colombia frente a las potencias extra-hemisféricas: el caso de las relaciones colombo-rusas ${ }^{* *}$
}

\author{
Colombia and Extra-Hemispheric Powers: the Case of \\ Colombo-Russian Relations
}

Colômbia face aos poderes extra hemisféricos: o caso das relações entre a Colômbia e a Russia

\footnotetext{
* Director del Centro de Investigaciónes CIES y Profesor de Tiempo Completo, Departamento de Estudios Políticos, Universidad Icesi, Cali, Colombia. El autor agradece el apoyo en la elaboración de este artículo a Manolo Constain.

** Este artículo se basa en parte de los hallazgos del proyecto de investigación N N. ${ }^{\mathrm{o}}$ 14.B37.21.0282 de 26/07/2012 del Ministerio de Educación y Ciencia de Rusia y la Universidad Icesi “La Modernización política de las regiones de Rusia". La versión anterior del artículo fue presentada en el Congreso Internacional "Rusia e Iberoamérica en un Mundo Globalizado: historia y perspectivas hacia el futuro" el 28 de septiembre de 2013 en la Universidad de San Petersburgo, Rusia.
}

Artículo de reflexión: recibido 08/09/2014 y aprobado 09/12/2014 



\title{
Resumen
}

El fin de la Guerra Fría dio inicio a un proceso de cambio con impacto en las relaciones internacionales en todo el mundo. En América Latina, una de las manifestaciones de este proceso es la creciente incidencia de las potencias extra-hemisféricas como China y Rusia. Sin embargo, en Colombia, los esfuerzos de la academia dirigidos a la construcción de un nuevo conocimiento sobre el tema han sido pocos. Una de las razones de esta escasez son las dificultades que surgen a la hora de analizar distintos aspectos de la política exterior colombiana hacia Rusia de la post-Guerra Fría usando las aproximaciones mainstream. Este artículo pretende reducir la brecha a través de la introducción de un marco de análisis que se fundamenta en la teoría del rol y que se usa para examinar el caso de las relaciones colombo-rusas durante el gobierno de Ernesto Samper Pizano (1994-1998). El autor señala que la aplicación de la teoría del rol en el análisis de la política exterior abre nuevas posibilidades de estudios de las relaciones de Colombia con los países desde fuera de las Américas.

Palabras clave: Política exterior colombiana, Política exterior rusa, Teoría del rol, Concepciones del rol nacional.

\begin{abstract}
In Latin America, the end of the Cold War was marked by the beginning of the important changes, including the growing incidence of extra-hemispheric powers like Russia. However, the academia has been rather slow in building new knowledge on the subject. One reason behind this scarcity is the difficulties that arise when attempting to examine various aspects of the Colombian post-Cold War foreign policy towards Russia using the so-called mainstream approaches. This article aims to narrow the gap by introducing a framework of analysis that is based on the role theory and is used to examine the case of the Colombo-Russian relations during the government of Ernesto Samper Pizano (1994-1998). The author argues that the application of the role theory to the foreign policy analysis opens new possibilities for studying Colombian relations with countries from outside the Americas.
\end{abstract}

Key words: Colombian foreign policy, Russian foreign policy, Role theory, National role conceptions.

\section{Resumo}

O fim da Guerra Fria assinalou o inicio de um processo de mudanças ressaltantes no que diz respeito às relações internacionais ao nível global. $\mathrm{Na}$ América Latina, uma das manifestações desse processo foi o acrescentamento da ocorrência de forças extra-hemisfé- 
ricas, como a China e Rússia. Na Colômbia, a academia não tem sido tão expedita para gerar os novos conhecimentos que permitam perceber o tema de maneira mais aberta, uma vez que, usando abordagens tradicionais, não é um processo simplista adiantar uma analise detalhada e compreensiva dos diversos aspectos que abrange a questão da política externa em relação à Rússia no período de pós-guerra fria. Este artigo tem o propósito de atravessar as lacunas conceptuais, e para tal fornece uma introdução de um marco de análise baseado na teoria da função, que é aproveitado para examinar o caso das relações Colombo-russas durante o governo do Dr. Ernesto Samper Pizano no período de 19941998. O autor ressalta o fato que a aplicação da teoria da função na análise da política externa, abre novas possibilidades para estudar o assunto das relações da Colômbia com países por fora das Américas.

Palavras-chave: Política externa da Colômbia, Política externa da Rússia; Teoria da função, Concepções das funções nacionais. 


\section{Introducción}

El estado actual de las relaciones internacionales a nivel global se caracteriza por la incertidumbre. Particularmente, en referencia al debilitamiento del papel que juegan en la arena internacional algunas potencias tradicionales, específicamente, con respecto al declive relativo de la hegemonía estadounidense y sus consecuencias globales y regionales. Este cambio obliga a los internacionalistas al análisis de los retos de un nuevo mundo en distintas dimensiones. Una de estas dimensiones destacadas tiene que ver con el fortalecimiento de unos actores internacionales que anteriormente gozaban de menor visibilidad y protagonismo, países como Rusia y China, potencias extra-hemisféricas cuyas voces en los asuntos del Hemisferio Occidental son cada vez más fuertes.

Los cambios mencionados: el declive relativo de la hegemonía estadounidense, y el fortalecimiento de las posiciones de algunos otros países, con sus propios intereses y estrategias que no necesariamente son obvios, sin duda crean grandes dificultades en la elaboración de estrategias de política exterior, tanto a mediano como a largo plazo, entre la mayoría de las potencias secundarias, incluyendo a Colombia, en los escenarios globales y regionales. Pero los cambios en las relaciones internacionales no solo generan desafíos, a su vez abren un abanico de oportunidades a quienes sepan aprovecharlas. Sobra decir que estas se presentan no solo para tomadores de decisiones, sino también para los investigadores internacionalistas: muchos de los que trabajan en el campo de relaciones internacionales usan los tiempos de incertidumbre para probar nuevas teorías e ideas, o al contrario, reviven el interés por teorías y marcos de análisis que todavía no gozan de un reconocimiento amplio en la disciplina para contribuir en el pensar sobre relevantes escenarios futuros de la transformación del mundo internacional.

En las últimas dos décadas se pueden describir varios momentos de las relaciones de Colombia con los países extra-hemisféricos; episodios que los investigadores académicos no consideraron suficientemente significativos para el análisis de la política exterior del país, o más bien: no consideraron otras herramientas analíticas para explicarlos. Para el autor de este artículo, uno de estos episodios es el sorprendente acercamiento que hizo el gobierno de Ernesto Samper Pizano (1994-1998) al gobierno 
de Rusia, en medio de una situación crítica en las relaciones de Colombia con los Estados Unidos; ocasionando una tensión en las relaciones entre Moscú y Washington mucho antes de la Guerra en Georgia en 2008 o la crisis en Ucrania en 2014, o sea, durante el periodo que normalmente se reconoce como de comportamiento cooperativo de Rusia con la política de Washington a nivel global (McFaul, 1998).

En este contexto, el presente trabajo tiene como objetivos centrales: primero, describir las experiencias de Colombia en su acercamiento a las potencias fuera de las Américas después del fin de la Guerra Fría, en particular, con Rusia, las cuales todavía no han sido examinadas de manera comprensiva por la literatura académica; y, segundo, demostrar la posibilidad de usar marcos de análisis distintos a los que se consideran como mainstream, en particular, la teoría del rol, para encontrar las claves de la actuación de los actores involucrados en los escenarios del pasado, y pistas para los posibles escenarios en el futuro de las relaciones de este país latinoamericano con las potencias tradicionales emergentes.

Las relaciones de Colombia con los Estados Unidos y sus vecinos han sido uno de los temas más estudiados por los internacionalistas con respecto a la política exterior de Bogotá (Bagley, 2000; Cardona, 2011; Drekonja, 1982; Pardo, 1997; Randall, 1992 y 2002; Sanchez y Monroy, 2012; Tickner, 2000 y 2008; y otros). Sin embargo, la importancia del tema escogido para este texto tiene que ver con el hecho de que todavía hay pocos esfuerzos para analizar las consecuencias que puede traer a Colombia el relativo retiro de los Estados Unidos de la región, en un contexto de creciente incidencia de las potencias emergentes extra-hemisféricas en países con los cuales las relaciones de Colombia han sido delicadas, como Venezuela y Nicaragua. Por otro lado, Colombia tiene que tomar nota sobre la cercanía que tiene la emergente potencia "nativa" de la región -Brasil- con Rusia, China e India, algunas de las más importantes potencias extra-hemisféricas en la región.

Desde el punto de vista de la seguridad nacional y la autonomía de la actuación de Colombia en la arena internacional, los retos centrales se enfocan en la identificación de las amenazas futuras y de las capacidades con las que cuenta el país para responder adecuadamente a ellas. En este contexto, hay que subrayar que los Estados Unidos sigue siendo la potencia política, 
económica y militarmente preponderante en el Hemisferio Occidental y a nivel global. No obstante, algunos países de la región han optado por utilizar la estrategia de soft balancing-entendida como búsqueda de cooperación relativamente prolongada y alterna a la militar- para contrabalancear las políticas unilaterales de la potencia superior en la región. En estas estrategias, el papel atribuido a las potencias extra-hemisféricas es clave (Flemes y Wojczewski, 2010). En el mismo tiempo, como argumentan Fabio Sanchez Cabarcas y Catalina Monroy Hernandez (2012: 334), los Estados Unidos siguen siendo el principal aliado geoestratégico de Colombia. No obstante, la continuidad de la asistencia militar y social de Washington a Colombia está condicionada a una serie de los factores que pueden alterar la capacidad de la respuesta estadounidense en los escenarios potencialmente críticos para Bogotá: los países vecinos de Colombia han logrado construir relaciones con potencias extra-hemisféricas que les permiten implementar políticas de soft balancing de manera bastante exitosa, mientras que la Casa de Nariño cuenta con pocas opciones.

A su vez, como va a demostrar este artículo, es difícil caracterizar los breves acercamientos de Colombia a Rusia durante el gobierno de Ernesto Samper como ejemplos de soft balancing; más bien son evidencias de otra índole: la premisa central de esta investigación consiste en que se trata de la manifestación de la selección de distintas concepciones acerca de roles nacionales realizada por los líderes de los países en cuestión, en el marco del proceso de la toma de decisiones en política exterior, lo que permitiría entender mejor algunos de los cambios inesperados en el comportamiento de los países, de otro modo difícilmente explicables como fue el acercamiento entre Bogotá y Moscú entre 1995 y 1998.

Este artículo consta de tres partes. La primera, comienza con la explicación acerca de la aplicación de la teoría del rol para el estudio de la política exterior y, en particular, para los casos latinoamericanos. Luego, usando la teoría del rol, se examina el caso de las relaciones entre Colombia y Rusia en la segunda mitad de la década del 90, para demostrar que mediante esta teoría es posible identificar las razones de un acercamiento inesperado para observadores domésticos e internacionales. El artículo concluye con una reflexión acerca de las posibilidades de construir marcos analíticos funda- 
mentados en la teoría del rol que permiten analizar las interacciones de los actores en la arena internacional, a veces difícilmente explicables desde la perspectiva ofrecida por otras teorías.

\section{La teoría del rol y los estudios de la política exterior: consideraciones iniciales}

La política exterior puede ser entendida como "la suma de las relaciones oficiales externas conducidas por un actor independiente (usualmente un Estado) en relaciones internacionales" (Hill, 2003: 3, citado por Velosa, 2012b: 40). Entendida así, la aplicación de la teoría del rol como marco de análisis conceptual se puede identificar en el campo de estudios internacionales desde hace mucho tiempo, aunque todavía no haya logrado constituirse en lo que puede llamarse el mainstream de la disciplina (Beneš, 2011). El término "rol" es una metáfora tomada del mundo del teatro, originalmente usada para explicar el comportamiento individual. Como destaca Cameron Thies (2009: 3), con el paso de tiempo el uso de la teoría del rol ha contribuido a la aparición de varias tradiciones (o versiones) teóricas. Esta diversidad fue posible gracias a que resultó viable aplicar la teoría del rol al estudio de la política exterior de manera similar a los estudios del compartimiento individual.

Específicamente, en el caso de la teoría internacional, se partió de las siguientes premisas. Primero, se puede decir que los líderes nacionales y miembros de élites son actores claves en el diseño de la política exterior. Son ellos quienes determinan, en última instancia, la concepción del rol nacional que va a jugar un país y cuál sería su actuación de acuerdo con el rol escogido (Holsti, 1970: 239-240). La otra premisa tiene que ver con el presupuesto de que el sistema internacional funciona como un sistema social estratificado, donde los líderes siempre están conscientes de las diferencias entre los países y actúan de acuerdo con las percepciones que tienen acerca de los roles que juegan estos países (Holsti, 1970; Walker, 1987). Sin embargo -y es la tercera premisa clave- hay una diferencia importante entre lo que pasa en una sociedad doméstica y los escenarios internacionales. En el primer caso, el papel de los otros es crucial en la definición de los roles individuales, pues en una sociedad doméstica se puede hablar sobre la existencia de un acuerdo sobre qué tipo de comportamiento se espera de los portadores de los roles particulares. En 
el caso de la sociedad internacional, caracterizada por su heterogeneidad, en muchos casos dicho acuerdo no existe, lo que puede conducir al crecimiento de la incertidumbre y de los conflictos en la base de lecturas distintas de los roles realizadas por los países mismos y por otros países.

Desde esta perspectiva, se puede pensar en el comportamiento de los países en la arena internacional como resultado de la ejecución de políticas cuyo fundamento son las concepciones de rol nacional escogidas por los líderes de esos países. Dichas concepciones están construidas con base en percepciones que mantienen los líderes con respecto a uno u otro aspecto de la política exterior e identidad de su país. Los tomadores de decisiones definen el rol de su país en un sistema internacional estratificado, en consecuencia, identifican las metas de la política exterior y diseñan las políticas que buscan hacer cumplir estas metas, en muchos casos, a través de la imposición de obligaciones sobre otros países con un rol inferior o subordinado; o, al contrario, rechazando los intentos de imponer las obligaciones de los otros (Holsti, 1970).

Como argumentan varios autores (Grossman, 2005; Thies, 2009; Velosa, 2012a; Walker, 1987), la ventaja de la teoría del rol en el estudio de la política exterior consiste en que esta se convierte en una herramienta analítica que permite resolver las dificultades del diseño de un marco de análisis apto para dar cuenta de distintos factores relevantes a la política exterior de uno u otro país: cuando un político define el rol internacional de su país, fundamenta su decisión en sus ideas acerca de las capacidades económicas, poder militar, riqueza, territorio o reconocimiento internacional, a través de un proceso donde pueden intervenir otras variables. En otras palabras, la teoría del rol pone las percepciones acerca de la concepción del rol nacional en el centro de todo el análisis, demostrando dónde y cómo surgen las preferencias con respecto a cambio y continuidad de la política exterior, expectativas e identificación de las opciones disponibles.

Teniendo en cuenta los argumentos presentados, se pueden pensar los escenarios de la arena internacional como resultado, por un lado, de los intentos de los líderes de los países de promover una concepción particular del rol nacional y, por otro lado, de la aceptación o el rechazo de esta concepción por parte de otros actores involucrados. También hay que destacar que cada país se puede caracterizar por múltiples roles asociados con su 
identidad, y que existe un cierto "repertorio" de roles entre los cuales los líderes y miembros de élites podrían escoger, dependiendo de las metas establecidas para su estrategia exterior en un momento dado. Normalmente, en dicho "repertorio" los roles son resultado de procesos e interacciones de larga duración, y reflejan las percepciones existentes que mantienen actores domésticos e internacionales (Beneš, 2011).

Desde esta perspectiva, los desacuerdos entre los países serían más probables en los escenarios donde existe un divorcio entre las concepciones de los roles nacionales que promueve un país y la percepción acerca de los mismos roles que tienen otros actores. De manera similar, las tensiones comienzan cuando algunos países intentan promover en el ámbito internacional una concepción particular del rol nacional de algún otro país, que no corresponde al rol que promueven su propio líder y sus élites. En consecuencia, los líderes y los miembros de élites de estos países buscan apoyo de otros actores que podrían fortalecer la concepción acerca del rol que les conviene. Las concepciones propias de los roles que quieren promover los otros países, el apoyo que consiguen los países "imponentes" por parte de otros actores, y la respuesta a la pregunta sobre qué tan exitosa, con respecto a las metas de su estrategia exterior, ha sido la imposición de concepciones de roles nacionales por parte de los países promotores, son factores claves que hay que tener en cuenta en el momento de acercarse a la tarea de analizar las tensiones entre actores internacionales como choques entre las percepciones acerca de los roles.

\section{Análisis de la política exterior en América Latina a partir de la teoría del rol}

Como se mencionó arriba, la teoría del rol no se ha utilizado para el análisis de la política exterior con tanta frecuencia como algunas otras aproximaciones. En aquellos estudios donde la teoría del rol sí fue llamada para construir marcos de análisis, dicha teoría se aplicaba casi exclusivamente al análisis de la política exterior de los países del centro, como los Estados Unidos y las potencias europeas, y algunos otros países como la Unión Soviética/Rusia y China. En América Latina escasean este tipo de trabajos. No obstante, recientemente se puede notar en la academia el surgimiento del interés con respecto a los estudios de la región para construir un marco de análisis de relaciones exteriores de países latinoamerica- 
nos a partir de la teoría del rol. En particular, los esfuerzos realizados por Eduardo Velosa Porras (2012b) y Cameron Thies (2014), hacen evidente el intento de los autores para acomodar a su análisis dos dimensiones del proceso relevantes a la investigación de la política exterior: el conocimiento general acerca de los factores que tienen su impacto en el diseño de la política exterior de los países latinoamericanos, y el conocimiento conceptual de la teoría del rol como una de las herramientas del análisis de la política exterior. En este artículo, y siguiendo la propuesta de Thies (2014), se combinan los acercamientos estructural y simbólico interaccionista para examinar los roles en tanto son posiciones dentro de un grupo organizado y una categoría de actores socialmente reconocida por la sociedad doméstica e internacional. De igual manera, es importante subrayar que este artículo comparte la premisa hecha por algunos autores (Stryker y Statham, 1985) de que se pueden reconocer simultáneamente los roles individuales y de los estados como la misma unidad de análisis.

Ahora, en cuanto las tradiciones académicas de análisis de la política exterior en América Latina, los investigadores (Hey, 1997; Thies, 2014) han identificado tres ejes de análisis: 1) pro centro vs. contra el centro; 2) autonomía vs. dependencia; y 3) enfoque económico vs. enfoque político. En el primero de los casos, se trata de los autores que están interesados en saber si los tomadores de decisiones están de acuerdo o son hostiles con respecto a las políticas que promueven las potencias dominantes en la arena internacional y las reglas de juego que estas potencias hacen cumplir a los otros actores debido a la dependencia económica, inestabilidad política doméstica, intereses de seguridad nacional e ideología estatal, entre otras razones. Desde esta perspectiva, en el caso del análisis de la política exterior colombiana, no cabe duda de que los estudios pioneros como lo de Gerhard Drekonja (1983) dan un fuerte impulso a esta línea de trabajos enfocados en las interacciones entre Bogotá y Washington. No obstante, y reconociendo la importancia de este tipo de acercamiento, tomado en su valor nominal, no siempre resulta suficientemente vigoroso para poder completar el cuadro de la política exterior colombiana, especialmente en casos como el analizado en este artículo: es muy poco probable que detrás de las interacciones del gobierno del presidente Samper con el gobierno ruso, hubiese un intento de reorientar, a largo plazo, los vínculos tradicionales de Colombia con los Estados Unidos. 
A su vez, los autores interesados en examinar las dimensiones de autonomía y dependencia intentan medir la capacidad de los lideres (y sus países) para diseñar e implementar la política exterior de manera autónoma con respecto a la incidencia de los actores internacionales o impacto de los desarrollos al otro lado de la frontera política internacional. Se toman, en primer lugar, tales factores como capacidades relativas de poder, los intereses de las potencias dominantes: en el caso de América Latina, fundamentalmente, los de los Estados Unidos (Sánchez y Monroy, 2012). Ahora, en cuanto el tercer eje, los estudios están centrados en temas relevantes a las ventajas y debilidades de la conducta de una política exterior específica desde la perspectiva de "lo práctico" (Tickner, 2008).

No es difícil notar, siguiendo a Hey (1997: 650-651) y Thies (2014: 3), que las antes mencionadas formas de analizar la política exterior se caracterizan por superponer las cuestiones de dependencia y autonomía. En muchos de los estudios de casos en América Latina, el poder explicativo está atribuido al análisis de las interacciones de los países de esta parte del mundo con Washington, donde este se considera como el caudillo internacional más importante en la definición de las dinámicas de interacciones internacionales en las Américas (Thies, 2014: 3-4). En consecuencia, desde la perspectiva de la teoría de roles, y hablando sobre líderes y países latinoamericanos, se pueden identificar concepciones de roles nacionales tales como caudillo, cliente, rivales, etc., en distintos escenarios que tienen que ver con el diseño de las estrategias de la política exterior.

Antes de continuar, es necesaria una mayor clarificación conceptual con respecto a la vinculación de la teoría de roles con el análisis de la política exterior. Velosa (2012b: 41) señala la importancia de tener en cuenta que: 1) la política exterior incluye todas las acciones del Estado frente a otros actores internacionales; 2) mientras su implementación puede hacerse en el interior del Estado, dichas acciones están relacionadas con la percepción [de los actores involucrados - V.R.] acerca de un escenario particular que debe ser atendido; 3 ) a pesar de que se puede identificar la incidencia de diversos actores en el diseño y la implementación de la política exterior, el papel central pertenece a quienes poseen capacidades institucionales para tal fin. Con respecto a esta última observación es fundamental subrayar que en el caso colombiano -así como en la mayoría de los países latinoamericanos- el ejecutivo posee un poder muy amplio para la implementación de la política exterior, en comparación con otros aspectos de 
la política nacional. Como muestra Milanese (2007), en los regímenes presidencialistas los ejecutivos están estructuralmente mejor posicionados que cualquier otro actor en el ámbito de política exterior, ya que cuentan con unas capacidades institucionales excepcionales que les facilitan controlar y utilizar los recursos de poder para establecer sus propias reglas de juego. En Colombia, ello se hace posible gracias a los arreglos institucionales establecidos por la Constitución Politica de 1991, que brindan al Presidente de la República un espacio de maniobra extenso en temas de política exterior, mientras que la incidencia de otros actores puede ser limitada (Ardila, Cardona y Tickner, 2002).

La existencia de elementos comunes con respecto al diseño e implementación de la política exterior por los líderes de los países latinoamericanos, hace posible que en este artículo el punto de partida sea el marco de análisis de la política exterior en América Latina que propone Thies (2014); y que tome en cuenta varias dimensiones de roles habitualmente ejecutados por los líderes de los países en esta parte del mundo.

Por promulgación del rol se hace referencia al grado del desempeño por un individuo de un papel, una vez seleccionado, en un entorno social particular. Cuestiones como la diversidad del repertorio del individuo, los esfuerzos en la ejecución del rol y el tiempo dedicado a este, son relevantes para el análisis de esta dimensión. Con lo anterior se ligan las expectativas del rol, que son las normas, creencias y preferencias relevantes al rendimiento del individuo con respecto a los roles asumidos por otros actores involucrados. Vale la pena enfatizar que entre estos se encuentran no solo los líderes de los países extranjeros, u otros actores al otro lado de la frontera nacional, sino también el auditorio nacional. Por esta razón Thies concluye que en América Latina las fuentes claves de las expectativas de rol son con frecuencia relacionadas con el prestigio personal y nacional y la presión de la opinión pública (2014: 7). Se puede también hablar sobre unas demandas del rol por las cuales se entiende la presión característica de un escenario específico con respecto a la selección de un rol particular. En este contexto, el auditorio juega papel de reality check y de una quía para el individuo con respecto al rol asumido.

Por último, pero no menos importante, es el concepto de la localización del rol que refiere al proceso internacional a través del cual el individuo elige un rol apropiado en un entorno específico. Para algunos investigadores como 
Walker (1979), la manera en que un gobierno conduce su política exterior es el resultado directo del proceso relevante a la localización del rol, mientras que para los otros como Thies (2012) la localización del rol se puede igualar a la socialización, en cuanto los nuevos miembros aprenden sus roles en respuesta a las señales y demandas del auditorio internacional. Para el autor de este texto -como se demostrará adelante- esta dimensión es particularmente importante para el análisis del caso de las relaciones colombo-rusas durante la presidencia de Samper, y las dinámicas de las relaciones entre Colombia y otras potencias extra-hemisféricas, pues el auditorio internacional podría disponer de los recursos necesarios para fortalecer otros aspectos en un escenario donde la disponibilidad de otros tipos de recursos está limitada y/o cuando otros actores intentan imponer los roles alternos al asumido por el líder del país.

No cabe duda de que una investigación que busca usar la teoría del rol para explicar los fenómenos internacionales debe, en primer lugar, identificar "el repertorio" de roles en la disposición de uno u otro líder y miembros de élite, así como tener en cuenta los procesos relevantes en la toma de decisión con respecto a la política exterior que habilitan o dificultan la imposición de uno u otro rol. Sin embargo, en términos de la metodología de investigación, difícilmente se puede hablar sobre la existencia de una vía acertada que permita identificar los roles de manera única. Se analizan principalmente los discursos de los líderes, las columnas de opinión y, cuando sea posible, se entrevistan a los líderes y miembros de élite. Por ejemplo, Holsti (1970) logra describir las concepciones de roles nacionales luego de examinar discursos, debates parlamentarios y declaraciones hechas en conferencias de prensa de más de setenta gobiernos en la segunda mitad de la década del 60 del siglo pasado. Otros se enfocan más en las entrevistas con líderes y miembros de las élites y otros actores relevantes. En el mismo tiempo, algunos otros investigadores hacen uso de otro tipo de datos: por ejemplo, Michael Grossman (2005), aparte de analizar los discursos del jefe del Estado ruso en la década del 90, prestó atención particular a los datos de la votación en la Organización de las Naciones Unidas para poder detectar el repertorio de roles relevantes a la política exterior rusa en esa década, e identificar el cambio de preferencias de la Rusia post-soviética y el surgimiento de tensiones con el Occidente relacionadas con el choque de roles. Para este artículo se optó por el análisis de fuentes primarias y secundarias, 
específicamente, entrevistas realizadas con el ex Presidente Ernesto Samper y a cuatro diplomáticos rusos (en este último caso asumiendo el compromiso de no mencionar sus nombres, debido a que algunos aspectos de las interacciones entre el gobierno colombiano y Moscú en la segunda mitad de la década del 90 permanecen bastante sensibles todavía para la política rusa en América Latina durante la segunda década del nuevo siglo), así como los discursos de Samper, y las declaraciones del Ministerio de Relaciones Exteriores de la Federación de Rusia, y el Departamento del Estado y sus funcionarios. También las columnas de opinión publicadas por los periódicos considerados portavoces de las élites políticas y empresariales como El Tiempo, en Colombia, Washington Post y New York Times, en los Estados Unidos, y Kommersant, en Rusia, resultaron de gran utilidad para la identificación de las concepciones de los roles principales de los líderes de los estados, existencia de repertorios y posibilidades de escoger entre roles. Es importante subrayar que la teoría del rol, cuando se usa como una herramienta del análisis de la política exterior, sostiene que todos los líderes tienen unas concepciones identificables del rol nacional para su país, cualquier sea el sistema político, la cultura política o el contexto regional particular.

\section{Las relaciones entre EE.UU. y Rusia en la mitad de la década del 90 desde la perspectiva de la teoría del rol}

Puesto que dos de los tres actores que nos interesan son los Estados Unidos y Rusia, antes de abordar el caso de las relaciones entre Bogotá y Moscú, identificamos las concepciones con respecto a sus roles nacionales durante la década del 90 del siglo pasado y en el comienzo del nuevo siglo. En el caso de los Estados Unidos, se puede identificar un repertorio muy amplio; pero, para la década del 90, los líderes y miembros de élite de este país destacaron y usaron los tres roles claves en los escenarios de esta investigación (Maull, 2011:169):

1. "Global Enforcer": los Estados Unidos tiene el derecho de intervenir en cualquier parte del mundo.

2. Promotor de principios demócratas y derechos humanos ("el democratizador"): los Estados Unidos promueve los regímenes democráticos y defiende globalmente los derechos humanos; están dispuestos a intervenir para facilitar la transición democrática en los países autoritarios y violadores de derechos humanos. 
3. Lider (de Occidente): los Estados Unidos cuenta con liderazgo entre los países de Occidente, son responsables de coordinar las políticas para enfrentar los retos de seguridad en los países occidentales.

En el mismo tiempo, se pueden destacar otras dos concepciones relevantes del rol nacional de los Estados Unidos: líder de las Américas, o sea, el país que define las reglas de convivencia en el continente, y líder de la lucha mundial contra el tráfico de drogas. ${ }^{1}$ El primer rol se evidencia a lo largo de la mayor parte de la historia política internacional en el Hemisferio Occidental. En cuanto el otro rol, en los discursos de los líderes de este país se subraya de manera constante que los Estados Unidos lideran los esfuerzos a nivel global en la lucha contra el tráfico ilegal de drogas, y que por esta razón tiene derecho, inter alia, a certificar a otros países en su contribución en esta lucha (Pardo y Cardona, 1995; Samper, 2013).

En el caso de Rusia, Grossman (1995: 343-345) identifica varios roles que intentaban promover Boris Yeltsin y los miembros de la élite rusa durante la década del 90. Para esta investigación nos interesan en particular:

1. Actor de alcance global: se propone que Rusia puede mantener relaciones con todo el mundo, sin limitar su influencia a las zonas geográficamente cercanas.

2. Mediador internacional: capacidad de Moscú para facilitar la resolución de conflictos internacionales.

3. Parte del mundo occidental: otra prioridad de la política exterior de Rusia es desarrollar los vínculos de cercanía y coordinación con los países del Occidente.

4. Actor internacional autónomo: énfasis con que Rusia podría conducir su política exterior de manera autónoma y partiendo de su entendimiento del interés nacional; tiene la ventaja de ser miembro fundador del Consejo de Seguridad de las Naciones Unidas.

5. Promotor del mundo multipolar: se declara el apoyo de Rusia a los procesos

1 Bruce Bagley (2013) resalta que en "los últimos veinte años, el narcotráfico ha sido una de las prioridades de las agendas y políticas de seguridad y defensa de Estados Unidos" (Bagley, 2013:47) y Arlene Tickner (2014) habla sobre el problema de "securitización de las drogas" destacando el carácter particular del tema para la política de Estados Unidos (Tickner, 2014: 26-27). 
de construcción de un mundo multipolar y de las reglas "más justas" en el sistema internacional.

6. Defensor de principios demócratas y derechos humanos: Rusia está dispuesta a promover los regímenes democráticos y a defender globalmente los derechos humanos.

Si se comparan las concepciones acerca de los roles nacionales promovidos por los Estados Unidos con las de Rusia en la década del 90, es fácil ver que algunos roles se pueden considerar complementarios: "promotor de principios democráticos y derechos humanos" (EE.UU.) vs. "defensor de principios democráticos y derechos humanos" (Rusia); "líder de Occidente" (EE.UU.) vs. "parte del mundo occidental" (Rusia). Mientras que otros podrían contribuir al surgimiento de tensiones y desacuerdos: "global enforcer" " (EE.UU.) vs. "actor internacional autónomo" y "promotor del mundo multipolar" (Rusia). Ambigüedad característica de las interacciones entre Moscú y Washington durante la primera década después del fin de la Guerra Fría.

Sin embargo, parece aún más interesante -desde la teoría del rol-analizar de qué manera el conflicto de los roles promovidos por los líderes y miembros de élite de las dos potencias (una tradicional, con el poder hegemónico en el Hemisferio Occidental, y otra emergente, proveniente desde fuera de las Américas) incide en las relaciones de la potencia hegemónica con un país como Colombia, recurso valioso que pudo ser utilizado por el líder del país latinoamericano para reducir el impacto de un rol indeseado que intentaron imponer los Estados Unidos a Colombia.

\section{Acercamiento del gobierno de Ernesto Samper (1994-1998) con la Federación de Rusia}

Durante los últimos años del gobierno de Gorbachov y los primeros años después la desaparición de la Unión Soviética, no hubo mayores cambios ni acontecimientos en las relaciones bilaterales entre Colombia y Rusia. En ese entonces, según la observación de un periodista, la sede de la Embajada colombiana en Moscú era "un plácido e invernal edificio con más pisos que funcionarios diplomáticos [...] se manejaban los asuntos a ritmo tan pausado como el de las relaciones Colombia-URSS” (Sierra, 1992). El gobierno de César Gaviria 
firmó, el 8 de abril de 1994, el Tratado sobre las bases de las relaciones entre la República de Colombia y la Federación de Rusia, que constituyó un reconocimiento mutuo de las nuevas realidades del mundo de la post-Guerra Fría, más que un breakthrough en las relaciones bilaterales.

La situación, sin embargo, cambió dramáticamente en la segunda mitad de la década del 90, al ritmo en que empeoraban las relaciones bilaterales entre el gobierno de Samper Pizano (1994-1998) y los Estados Unidos de América. Este retroceso se debía a las acusaciones contra el electo primer mandatario colombiano de tener vínculos con el cartel de Cali.

Arlene Tickner (2000:44) propone una periodización de las relaciones bilaterales entre Colombia y los Estados Unidos durante la época de Samper que es útil para una mejor comprensión de las dinámicas de las relaciones entre Rusia y Colombia en el mismo periodo. Según Tickner, se pueden distinguir tres fases distintas de las dinámicas de las relaciones colombo-estadounidenses. La primera, entre agosto de 1994 y agosto de 1995: "la relación de trabajo", cuando Washington, a pesar de sus crecientes sospechas sobre los vínculos del presidente Samper con el mundo del narcotráfico, mantenía la colaboración con el nuevo gobierno en Bogotá. La segunda, entre septiembre de 1995 y febrero de 1997: la etapa del no reconocimiento, cuando "el debilitamiento del Presidente colombiano [...] se convirtió en el objetivo principal de algunos funcionarios del Departamento de Estado" (Tickner, 2000: 46), mediante la descertificación de Colombia en su lucha con el narcotráfico y la revocación de la visa de Samper después de que el presidente colombiano fuera absuelto por el Comité de Acusaciones de la Cámara de Representantes. La tercera sería una gradual normalización de las relaciones, entre marzo de 1997 y agosto de 1998.

Estos sucesos pueden ser interpretados como la imposición de distintos roles sobre Colombia en la arena internacional: 1) miembro respetado de la comunidad internacional y colaborador en la lucha contra las drogas ilícitas (Clinton, 1995); 2) "narco democracia" que no colabora con los esfuerzos por combatir el tráfico de drogas (El Tiempo, 12 de julio de 1996); 3) miembro de la comunidad americana dispuesto a colaborar con los Estados Unidos (Committee on International Relations, 1998).

Desde la teoría del rol, la respuesta de Colombia se relacionaría con el surgimiento de las concepciones de roles que ayudan a construir una percepción 
diferente a los roles impuestos por Washington. Analizando los discursos del presidente colombiano y las columnas de opinión publicadas entre 1994 y 1998, así como la entrevista realizada al primer mandatario por el autor de este texto, se pueden identificar al menos dos roles que el líder colombiano intentaba promover en ese entonces:

1. Actor internacional autónomo: a pesar de los intentos de los Estados Unidos para aislar a Colombia en la comunidad internacional, el país puede construir relaciones con los miembros plenamente reconocidos en la arena internacional de manera autónoma e independiente (Samper, 1995).

2. Lucha contra el tráfico de drogas: a pesar de la descertificación del país, Colombia lidera la lucha contra el tráfico ilegal de drogas y cuenta con la colaboración de actores internacionales (Samper, 1996).

La sorprendente activación de las antes "dormidas" relaciones entre Colombia y Rusia, en la segunda parte de la década del 90, tiene que ver precisamente con el deterioro de las relaciones de Samper con EE.UU. durante la segunda y la tercera fases de la periodización mencionada y con la imposición de roles. El escenario es que el entonces primer mandatario colombiano, debilitado y prácticamente excluido por los estadounidenses de la discusión de asuntos tan importantes de la política exterior como los relacionados con la política antinarcóticos de los Estados Unidos, intenta aproximar a Colombia (una potencia secundaria) a Rusia, una ex superpotencia que no obstante estar en proceso de recuperar su rol en la arena internacional, contaba con un legado significativo en las políticas internacionales $^{2}$, además de mantener una presencia clave en las instituciones internacionales y constituir una alternativa viable como proveedor de armamentos. Rusia ya gozaba, en el contexto internacional, del reconocimiento de algunos de sus roles, en particular, como actor internacional autónomo que comenzaba a causar algunas tenciones con la concepción de la rol nacional percibida por Washington con respecto a Moscú.

2 Es indicativo que en la conversación personal con el autor de este artículo Ernesto Samper (2013b) se referiría a la "Unión Soviética", aunque, por supuesto, era consciente de que se trataba de la Rusia de la época post-soviética. 
Table 1. Colombia, Rusia y Estados Unidos 1994-1998

\begin{tabular}{|c|c|c|c|}
\hline $\begin{array}{l}\text { Relaciones } \\
\text { entre Colombia } \\
\text { y Rusia }\end{array}$ & $\begin{array}{l}\text { Relaciones } \\
\text { "dormidas" }\end{array}$ & $\begin{array}{l}\text { - Oct. 1995: visita de } \\
\text { Soskovetz } \\
\text { - Mar. 1996: firma del } \\
\text { convenio de cooperación } \\
\text { técnica militar entre } \\
\text { Colombia y Rusia } \\
\text { - Oct. 1996: compra de } \\
\text { helicópteros rusos }\end{array}$ & $\begin{array}{l}\text { Nov. 1997: visita de } \\
\text { Primakov a Bogotá, } \\
\text { firma del acuerdo } \\
\text { de cooperación } \\
\text { en la lucha contra } \\
\text { el tráfico ilegal de } \\
\text { drogas }\end{array}$ \\
\hline $\begin{array}{l}\text { Relaciones } \\
\text { entre Colombia } \\
\text { y EE.UU. }\end{array}$ & $\begin{array}{l}\text { "Relación de } \\
\text { trabajo" }\end{array}$ & $\begin{array}{l}\text { "No reconocimiento" y } \\
\text { descertificación de Colombia } \\
(1996,1997)\end{array}$ & $\begin{array}{l}\text { "Búsqueda de } \\
\text { normalización" } \\
\text { Certificación de } \\
\text { Colombia (1998) } \\
\end{array}$ \\
\hline $\begin{array}{l}\text { Administración } \\
\text { de Ernesto } \\
\text { Samper }\end{array}$ & $\begin{array}{l}\text { Ago. } 1994 \text { - } \\
\text { Ago. } 1995\end{array}$ & Sept. 1995 - Feb. 1997 & $\begin{array}{l}\text { Mar. } 1997 \text { - Ago. } \\
1998\end{array}$ \\
\hline
\end{tabular}

Fuente: elaboración propia a partir de Tickner (2000).

Este último punto resultó ser uno de los factores claves en las relaciones entre el gobierno de Samper y Moscú en la segunda mitad de la década del 90. En ese entonces, el complejo militar industrial ruso comenzó a actuar como un rival importante de las industrias militares estadounidenses en el mercado internacional; según varios informes del Instituto Internacional de Estudios para la Paz de Estocolmo -SIPRI- publicados a finales de la década en cuestión (SIPRI 1998, 1999). Las industrias militares rusas mostraron interés en aumentar su presencia en las regiones de antigua influencia soviética, así como de encontrar nuevas oportunidades en los mercados que estaban "cerrados" en la época de la Guerra Fría: en estos años los rusos promovían negocios de armas en América Latina. La visita a Colombia del vice-primer ministro ruso Oleg Soskovetz, en octubre de 1995, es indicativa del creciente interés de algunos sectores del establecimiento ruso por explorar las nuevas oportunidades en Colombia ${ }^{3}$.

3 Oleg Soskovetz era el encargado en el gobierno de Rusia de los asuntos de la industria militar. En 1995, era considerado el segundo más poderoso de la política rusa y posible sucesor de Boris Yeltsin. Durante su visita a Colombia, Soskovetz discutió con el Presidente Samper las perspectivas de la cooperación ruso-colombiana (El Tiempo, 18 de octubre de 1995). 
En este contexto, es entendible la importancia que Samper pudo atribuir al convenio para la cooperación técnica-militar entre Rusia y Colombia, que entró en vigencia el 13 de marzo de 1996, apenas dos semanas después de que Colombia fuera descertificada por los Estados Unidos 4 . Algunos medios de comunicación del país vincularon directamente el convenio ruso-colombiano con el empeoramiento de las relaciones entre Colombia y los Estados Unidos: para El Tiempo (14 de marzo de 1996), el objetivo del acuerdo era "amortiguar los posibles efectos que la descertificación traería al país". Desde el punto de vista de la seguridad nacional -y la promulgación de la concepción del rol del actor autónomo-, en caso de que los Estados Unidos aplicaran sanciones, Colombia tendría alternativas viables para la compra de armas.

A través del convenio con Rusia, el presidente colombiano daba muestras a Washington de que estaba dispuesto a considerar escenarios alternativos a las tradicionales relaciones bilaterales entre Colombia y los Estados Unidos, en el caso de que persistiera el rechazo estadounidense de su papel en las relaciones bilaterales y la imposición del rol negativo al líder colombiano. Desde la perspectiva de la teoría del rol, al presidente colombiano se le reducían los espacios de maniobra: ante la presión que los Estados Unidos ejercían, acercarse a Rusia era una opción disponible para cumplir con las expectativas de su rol como líder de un país autónomo en la arena internacional. Por demás, este acercamiento era una respuesta al llamado del nuevo liderazgo ruso en la arena internacional como actor de alcance global y autónomo.

Desde esta perspectiva, se puede constatar que la estrategia de Samper funcionó, por lo menos hasta cierto límite. En el ámbito diplomático, Rusia fue uno de los pocos países que tras la absolución del Presidente Samper por parte de la Cámara de Representantes del Congreso colombiano declaró su respeto a la decisión, mientras que los Estados Unidos revocaron la visa a Samper. Rusia criticó a los Estados Unidos "por apropiarse del dere-

4 Según Pardo y Cardona (1995), el proceso de certificación "se trata de una decisión unilateral de los Estados Unidos, y más concretamente del Ejecutivo. Por razones de política interna el país tiene establecidos mecanismos legales, por medio de los cuales algunas instancias del Gobierno y del Congreso desean conocer la opinión que el presidente y su equipo tienen cada año sobre los esfuerzos que un número determinado de países están efectuando en diversas materias." 
cho a juzgar a otros". El Kremlin optó incluso por "señalar que Colombia, como Estado soberano, tomó esta decisión en plena concordancia con el procedimiento constitucional"; y decidió "expresar su inquietud por la reacción de Estados Unidos" que "no se la puede reconocer aceptable por su tono ni por su contenido duro y arrogante", "del estilo de la Guerra Fría”. (El Tiempo, 27 de junio de 1996).

Es probable que las declaraciones del Ministerio de Relaciones Exteriores de Rusia tuvieran poco impacto inmediato en las relaciones bilaterales entre Colombia y los Estados Unidos, pero fueron utilizadas por Samper para demostrar a la opinión pública colombiana la existencia de apoyo en la comunidad internacional a su papel como Jefe del Estado frente al rechazo estadounidense. No obstante, los acontecimientos posteriores en las relaciones entre Rusia y Colombia, que examinaremos en breve, en particular la decisión del gobierno de Samper de comprar helicópteros militares rusos en 1996, y la visita a Colombia del Ministro de Relaciones Exteriores de Rusia Yevgeny Primakov en noviembre de 1997, sí pudieron despertar algunas alarmas en Washington, porque coincidieron con otros hechos en la arena internacional: los acercamientos de Rusia con países como Irán y Corea del Norte que, muy pronto, tenían una continuación en un acto simbólicamente importante: Primakov canceló su visita a los Estados Unidos en protesta por la actuación unilateral de los Estados Unidos en la antigua Yugoslavia, cuando ya su avión sobrevolaba el océano Atlántico rumbo a Washington.

\section{La conexión ruso-colombiana}

Vale la pena subrayar que el acercamiento de Samper a Rusia ocurría en un contexto hemisférico particular: se comenzaba a hablar en voz alta sobre la presencia fuerte del crimen organizado ruso en América Latina y el Caribe. Estos desarrollos, sin duda, tenían un impacto negativo sobre las concepciones de roles nacionales de Colombia y Rusia. Según Bruce Bagley: "los vínculos o 'alianzas estratégicas' entre varios grupos del crimen organizado ruso y las organizaciones criminales transnacionales en América Latina y el Caribe, ya eran substanciales y pueden ser caracterizadas por una expansión rápida" (2004: 261). En el caso colombiano, ya desde la primera parte de la década 90, los grupos del crimen organizado ruso en Norte América co- 
nectados con sus homólogos en Rusia habían establecido contactos con los narcotraficantes colombianos para la adquisición de cocaína y la venta de armamento a los carteles y la guerrilla colombianos ${ }^{5}$.

No hay duda de que las autoridades estadounidenses estuvieron preocupadas por la existencia de una conexión criminal ruso-colombiana. La publicación de un informe especial del Washington Post en septiembre de 1997 refleja con claridad esta preocupación ${ }^{6}$. Por otro lado, recordamos que Samper estaba excluido, por iniciativa estadounidense, del manejo de la política antinarcóticos con los Estados Unidos; como se mencionó anteriormente, tenía poco espacio de maniobra. En este entorno, el presidente optó por acercarse a Rusia para presionar un cambio de actitud de los tomadores de decisiones en Washington; los cuales, obviamente, estuvieron interesados no solo en el debilitamiento de los vínculos entre el crimen organizado ruso y los narcotraficantes colombianos, sino también en la promoción de los intereses estadounidenses en otros aspectos de las relaciones bilaterales con Colombia. El caso de la venta de los helicópteros rusos al Ejército colombiano se puede considerar una evidencia a favor de esta afirmación.

La importancia que Rusia estaba atribuyendo a las ventas de sus helicópteros en América Latina, no solo en términos de las ganancias económicas sino también como herramienta para el fortalecimiento de la percepción sobre su rol como actor fuerte y de alcance global, se evidencia, por ejemplo, por la participación en la promoción de los aparatos rusos en el mercado latinoamericano de algunos "pesos pesados" como el expresidente de la Unión Soviética Michael Gorbachov, quien visitó varias exhibiciones de la producción del sector aeronáutica rusa (Trushin, 1998). De igual manera, para Rusia era entendible que sin

5 En febrero de 1993, las autoridades rusas confiscaron cerca de San Petersburgo una tonelada de cocaína camuflada en carne enlatada. Este caso fue el primero de varios en los que se pudo vincular al narcotráfico colombiano, pero no fue investigado debido a "la ubicación de los sospechosos fuera del país". En los 1990, en Rusia, se preocuparon ante la perspectiva de convertirse en punto clave del tránsito de drogas desde Suramérica a Europa. (Paoli, 2002: 31).

6 El periodista Douglas Farach identificó cinco dimensiones claves de la "conexión rusa": 1) Más de 30 grupos del crimen organizado operaron desde Miami, Nueva York y Puerto Rico en negocios de cambio de armas por cocaína; 2) Los grupos de crimen organizado en Rusia que controlaron las antiguas empresas estatales soviéticas de producción de armas, suministraron armamentos a los grupos al margen de la ley en Colombia por vía marítima, utilizando puertos en la costa pacífica colombiana; 3) Las armas se cambian por cocaína refinada producida por los carteles en Colombia; 4) La cocaína termina en los mercados europeos; 5) Los grupos criminales rusos abrieron cuentas de manejo electrónico en bancos del Caribe para el lavado de dinero (Farach 1997). 
apoyo político de más alto nivel no lograría efectuar las ventas deseadas ${ }^{7}$. Cuando el Ministerio de Defensa colombiano anunció el otorgamiento del 40 por ciento de la licitación internacional de la compra de helicópteros a Rusia, esto se consideró un avance significativo de la política exterior del gobierno ruso no solo en materia de cooperación técnica-militar, sino también en el ámbito político internacional, como un reconocimiento del nuevo rol internacional de Rusia de alcance verdaderamente global (Bulavinov, 1997).

En el contexto en que se desarrollaba el conflicto entre Samper y el gobierno estadounidense, no sorprende el hecho de que la venta de los helicópteros generase una reacción negativa en Washington. El subsecretario de Estado para Asuntos Antinarcóticos, Robert Gelbard, manifestó que el Gobierno estaba "profundamente decepcionado y sorprendido" por la compra de helicópteros rusos por el gobierno colombiano (El Tiempo, 30 de octubre de 1996). ${ }^{8}$ Luego del pronunciamiento de Gelbard, hubo un intercambio de declaraciones entre Moscú y Washington, en un tono no muy diplomático y atípico para las relaciones entre la Casa Blanca y el Kremlin. El portavoz del Ministerio de Relaciones Exteriores ruso "recordó que Gelbard había amenazado descaradamente a las autoridades colombianas con un empeoramiento de las relaciones entre EE.UU. y Colombia", y que tal declaración constituía "un intento de injerencia en las relaciones comerciales de dos Estados soberanos". Para Moscú, la reacción del diplomático estadounidense al acuerdo comercial tenía "carácter marcadamente político e inamistoso" con respecto a Rusia y Colombia; el Kremlin consideró el pronunciamiento de Washington "como una descarada violación de las normas y principios universales de libertad del comercio internacional y de la competencia legal" (El Tiempo, 30 de octubre de 1996). Es importante subrayar que, aparentemente, en ningún otro caso en el subcontinente latinoamericano se dio nada parecido a este cruce de palabras entre Moscú

7 Las compañías rusas iniciaron su conquista del mercado nacional colombiano a principio de la década del 90 y habían efectuado ya varias ventas del sector aeronáutico ruso antes de ganar la discutida licitación. Así pues, en el caso de las ventas de helicópteros, Rusia consideraba el mercado colombiano como la base de una posible expansión de sus ventas y servicios de mantenimiento en la región.

8 Vale la pena notar que la decisión del gobierno de Colombia se dio a conocer sabiendo que los Estados Unidos estaban dispuestos vender sus helicópteros a Colombia a pesar de que el país no fuera certificado. (United States Congress, 1996). 
y Washington en octubre de 1996, usando el vocabulario propio de los tiempos de la Guerra Fría, una década antes de que se comenzara a hablar de una "Nueva Guerra Fría".

La otra ocasión que demuestra que la estrategia escogida por el jefe del gobierno colombiano para acercar Colombia a Rusia contribuyó en el cambio de las políticas estadounidenses hacia Colombia, tiene que ver con la mencionada "conexión rusa". Mientras Colombia debía aceptar, bajo la presión estadounidense, la creación de un grupo de trabajo conformado por los ministros de Relaciones Exteriores, Defensa y Justicia que excluía al propio presidente Samper (Tickner 2000: 47), el primer mandatario colombiano optó por ofrecer a Rusia su cooperación en la lucha contra el narcotráfico: el acuerdo correspondiente fue firmado durante la visita del Ministro de Relaciones Exteriores de Rusia, Yevgeny Primakov a Bogotá el 26 y 27 de noviembre de 1997, abriendo las puertas al intercambio de información y a medidas conjuntas entre los dos países sin la participación directa de los Estados Unidos. Cuando Primakov arribaba a la capital colombiana, el país no fue certificado como socio de los Estados Unidos en la guerra contra las drogas por segunda vez consecutiva. No obstante, tenía que implementar una agenda específica de acuerdo a las indicaciones de las autoridades estadounidenses (Tickner 2000: 47). En el caso de la cooperación con Rusia en la lucha con el narcotráfico transnacional, el presidente Samper fue el protagonista en un tema de vital interés para los Estados Unidos, fortaleciendo el rol de Colombia como luchador contra el tráfico de drogas.

Sería equivocado atribuir el comienzo de los cambios de las políticas estadounidenses hacia Colombia, evidenciado por la certificación del país en marzo de 1998 (tercera fase de la periodización de Tickner: "la normalización gradual"), únicamente al acercamiento colombiano a Rusia. Los Estados Unidos tenían que tomar en consideración varios factores y el hecho de que sus políticas en el país sudamericano resultaron contraproducentes era clave. Pero tampoco se puede negar la incidencia que tuvo en la decisión de dar nuevo rumbo a su política hacia Bogotá, la creciente preocupación en Washington

9 Los comentadores internacionales comienzan a hablar sobre una nueva guerra fría entre Rusia y Occidente en 2007, luego del discurso pronunciado por el presidente Vladimir Putin en la Conferencia de Múnich sobre política de seguridad. 
sobre el fortalecimiento de las relaciones ruso-colombianas. El apoyo diplomático ruso al presidente colombiano no fue grato a los Estados Unidos; la sorprendente pérdida del contrato en materia tan sensible como la venta de armas y la firma de un convenio que los excluía sobre cooperación en otra área de extrema importancia como la lucha contra el narcotráfico, donde los Estados Unidos estuvieron proyectando de manera constante la concepción del rol nacional estadounidense como el líder global contra el tráfico ilegal de drogas, no se ignoró en la Casa Blanca.

Es cierto que la visita de Primakov generó muchas especulaciones, sobre todo en medios de comunicación latinoamericanos, acerca del surgimiento de una nueva política rusa hacia América Latina (Rouvinski, 2014). Pero el hecho de que Rusia estuviera dispuesta a aceptar el acercamiento de Colombia durante el gobierno de Samper, tiene otra explicación. Por un lado, el escenario bilateral entre Colombia y los Estados Unidos se prestó para exponer las nuevas retoricas del Kremlin que buscaban el fortalecimiento de una nueva concepción sobre el rol de Rusia en el sistema internacional. Por otro lado, el repertorio de los roles en Moscú en ese entonces también incluía la concepción nacional de Rusia como el país que colabora con los Estados Unidos. El presidente ruso Boris Yeltsin usaba distintos roles para poder balancear entre dos campos de las elites rusas: uno -al que pertenecía Primakov- que optaba por el distanciamiento con Occidente; y el otro, que deseaba un acercamiento de Rusia con los Estados Unidos y los países europeos (McFaul, 1998; Trenin, 2007). Las restricciones de esta índole, en términos de localización de roles, redujeron significativamente el espacio de maniobra para Primakov. Esta es la respuesta a la pregunta sobre por qué los planes más ambiciosos, con respecto al futuro político de las relaciones colombo-rusas, no fueron puestos en práctica ${ }^{10}$.

10 En conversaciones del autor de este artículo con algunos funcionarios del Ministerio de Relaciones Exteriores de Rusia durante el gobierno de Samper, se mencionó que la Embajada Rusa recibió una propuesta de los líderes de las FARC para que el gobierno de Rusia fuera intermediario en las posibles negociaciones de paz. Se discutió también la posibilidad de que Samper realizara una visita estatal a Moscú. Hay evidencias de que Washington tenía que ejercer presión directa sobre Moscú para que la visita no se realizara. 


\section{Conclusiones}

El artículo empezó refiriéndose a la incertidumbre característica del estado actual de las relaciones internacionales. Desde la perspectiva de la teoría del rol, entre las razones que causaron el surgimiento de esta incertidumbre se pueden nombrar las crecientes diferencias entre las percepciones acerca de los roles nacionales que sostienen los actores tradicionales, y los cambiantes puntos de vista sobre dichos roles de los actores que anteriormente gozaron de menor incidencia en la arena internacional. Puesto que se puede considerar al sistema internacional como un sistema estratificado, con frecuencia los intentos de los líderes se dirigen hacia la ampliación y/o cambio de los repertorios de los roles, y terminan en el surgimiento de tensiones como consecuencia del rechazo al cambio por otros miembros de la comunidad internacional. En América Latina, este proceso tiene lugar en un contexto de continuación del debate tradicional acerca de las cuestiones de dependencia y autonomía, pero con una diferencia importante: desde el fin de la Guerra Fría, potencias extra-hemisféricas como China y Rusia muestran mayor interés en buscar el reconocimiento de las nuevas concepciones de sus roles nacionales por los países latinoamericanos, mientras que estos consideran la novedad como la oportunidad de fortalecer concepciones propias.

En este sentido, el caso del acercamiento que tuvo el Presidente Samper con los líderes de Rusia en la segunda mitad de la década del 90 es particularmente interesante. Por un lado, permite al investigador entender mejor, desde una perspectiva conceptual, los aspectos de la política exterior relacionados con la promulgación de la concepción del rol nacional, las expectativas del rol en relación con los temas del prestigio personal y nacional, las demandas del rol y su localización. Por otro lado, el análisis del caso puede indicar pistas para la búsqueda de una respuesta adecuada a cuestiones tales como, por ejemplo, por qué las dinámicas de las interacciones políticas entre Colombia y China han sido bajas en comparación con otros actores de la región (Borda y Berger 2012).

En su dimensión internacional, la presidencia de Ernesto Samper estuvo marcada por una crisis en las relaciones con los Estados Unidos. Muy pronto después de ocupar el cargo, su desempeño en calidad de Jefe de Estado autónomo comenzó a cuestionarse en la arena internacional como resultado de la imposición de una concepción del rol nacional de Colombia, formulado por la 
Casa Blanca, que en el entorno social particular de entonces no pudo ser aceptada ni por Samper ni por la opinión doméstica colombiana. Desde la perspectiva de roles, la doble descertificación de Colombia estuvo ligada con las creencias vinculadas con las expectativas del rendimiento de Samper como presidente de la República, con respecto a normas y reglas de juego anteriormente acordados entre Bogotá y Washington. En el mismo tiempo, se pueden identificar los elementos de la agenda política nacional colombiana en la década del 90 que son las evidencias de la existencia de una demanda asociada con el rol del Ejecutivo en relación con el tema del prestigio individual y nacional. En este escenario, se comenzó la búsqueda de una nueva localización del rol entendida como una socialización entre los miembros de la comunidad internacional. Finalmente, se encontró una respuesta afirmativa proveniente desde Moscú.

En el caso anteriormente explicado, hay que notar que el liderazgo ruso tenía sus propios intereses para apoyar al gobierno de Samper, los cuales estaban relacionados con las tareas de promulgación y localización de una nueva concepción del rol nacional ruso. Esta concepción todavía no era dominante, pero ya hacía parte del repertorio de los líderes: Rusia como actor global y autónomo. En otras palabras, las dos partes involucradas se necesitaban una a otra para implementar las tareas de la promulgación de las concepciones de rol nacional que les convenían.

El escenario cambia rápidamente con la llegada del gobierno de Andrés Pastrana Arango (1998-2002) y sobre todo durante los dos gobiernos de Álvaro Uribe Vélez (2002-2010), cuando las tareas de promulgación de los roles nacionales de Colombia y de Rusia dejan de ser complementarias y la colaboración entre los dos países nunca llega a los niveles alcanzados durante la presidencia de Ernesto Samper. En este sentido, no es difícil ver que, desde la perspectiva de la teoría del rol, las poco dinámicas relaciones políticas actuales entre Colombia y la potencias extra-hemisféricas, en primer lugar Rusia y China, se explican por la falta de la complementariedad de las tareas relevantes a la promulgación de roles, y las dificultades en cuanto la localización de la nueva concepción de los roles nacionales de estos países en Colombia. Como muestra Armony (2012) para el caso chino, Beijing todavía no ha logrado construir una identidad acertada que sería aceptada por los tomadores de decisiones en Colombia y que permitiría alcanzar un 
grado mayor de reconocimiento con respecto a China y su agenda de política exterior, como es el caso de algunos otros países de la región.

Los resultados de la investigación presentados en este artículo señalan que la teoría del rol puede ser muy útil para la construcción de marcos de análisis de la política exterior, especialmente con referencia a los casos donde existen dificultades para explicar la conducta de los actores desde otras perspectivas y aproximaciones. De igual manera, planteando el estudio de la concepción de roles nacionales, y reconociendo como eje de la discusión la existencia de ciertos repertorios de roles, se logran explicar los cambios inesperados de la política exterior, identificando los mecanismos empleados para su implementación, fuentes de preferencias, fortalezas y debilidades, y eventualmente anunciando los futuros escenarios con mayor precisión.

\section{Referencias}

Ardila, M., Cardona D. y Tickner A. (2002). Prioridades y desafíos de la politica exterior colombiana. Bogotá: Friedrich Ebert Stiftung, Colombia

Armony, A. (2012). A View from Afar: How Colombia Sees China, China Quaterly, 209: 178-197

Bagley, B. (2000). Narcotráfico, Violencia Política y Política Exterior de Estados Unidos Hacia Colombia en los Noventa. Colombia Internacional, No. 49-50, 5-38.

(2004). "Globalization and Transnational Organized Crime:

The Russian Mafia in Latin America and the Caribbean" en Menno Velinga, ed., The Political Economy of the Drug Industry: Latin America and the International System, Gainesville: University Press of Florida, 261-296.

(2013). Principales tendencias del siglo XXI en cuanto al crimen organizado, el narcotráfico y la democracia en la región. Trans-Pasando Fronteras, (3), 47-54.

Beneš, V. (2011). Role Theory: A conceptual framework for the constructivist foreign policy analysis? Paper presented at the Third Global International Studies Conference "World Crisis. Revolution or Evolution in the International Community?", 17-20 August 2011, University of Porto, Portugal

Borda Guzmán, S. y Berger M. P. (2012). Relaciones bilaterales China y Colombia: 1990-2010, Colombia Internacional, 75, 83-129. 
Bulavinov, I. (1997). Rosvooruzhenie toma a América Latina en los helicópteros Mi-17 [en Ruso]. Kommersant. (61), 1.243.

Cardona D. (2011). Introducción: ¿Puede tener Colombia una estrategia de política exterior? Cardona, D. (Ed.) Colombia: una politica exterior en transición. Bogotá: Fundación Friedrich Ebert en Colombia (Fescol), xvi- xli.

Clinton, W. (1995). Memorandum on Narcotics Producing and Transit Countries. February 28. Washington. DC: The White House

Committee on International Relations. (1998). U.S. narcotics policy toward Colombia: hearing before the Committee on International Relations, House of Representatives, One Hundred Fifth Congress, second session, February 26. Washington, D.C.: US Government Printing Office

Drekonja, G. (1983). "Formulando la Política Exterior Colombiana" en: Drekonja, G. y Tokatlián, J. (Eds.) Retos de la política exterior colombiana. Bogotá: Fondo Editorial Cerec, 59-97.

El Tiempo (18 de octubre 1995), Viceministro ruso ofrece vender armas a Colombia.

(14 de marzo 1996). El convenio militar entre Rusia y Colombia. (27 de junio 1996). Rusia respeta absolución del presidente Samper.

(12 de julio 1996). Dos años de guerra fría entre Colombia y Estados Unidos.

(30 de octubre 1996). Rusia critica injerencia de E.U. en comprar de helicópteros.

(11 de marzo 1998). Rusia ofrece intermediar con guerrilla.

Farah, D. (1997). Russian Mob, Drug Cartels Joining Forces. Washington Post (29 September 1997).

Flemes, D. y Wojczewski, T. (2010). Contested Leadership in International Relations: Power Politics in South America, South Asia and Sub-Saharan Africa, GIGA (German Institute of Global Area Studies), WP 121/2010. Recuperado http://dx.doi.org/10.2139/ssrn.1547773

Grossman, M. (2005). Role Theory and Foreign Policy Change: The Transformation of Russian Foreign Policy in the 1990s. International Politics, 42, 334-351.

Hey, J. (1997). Three Building Blocks of a Theory of Latin American Foreign 
Policy. Third World Quarterly 18, 631-657.

Holsti, K. (1970). "National Role Conceptions in the Study of Foreign Policy". International Studies Quarterly, 14(3): 233-309.

Maull H. (2011). "Hegemony Reconstructed? America's Role Conception and its "leadership" within its core alliances" in Sebastian Harnisch, Cornelia Frank, Hanns W Maull (Eds.) Role Theory in International Relations. New York: Routledge, 167-193.

McFaul, M. (1998). A Precarious Peace: Domestic Politics in the Making of Russian Foreign Policy. International Security, 22 (3), 5-35.

Milanese, J.P. (2007). Colombia y Venezuela: interpresidencialismo e integración regional. Cali: Universidad Icesi.

Pardo, R. (1997). "Colombia y Estados Unidos, intoxicados por las drogas" en Ramírez, Socorro y Restrepo, Luis Alberto, (Eds.) Colombia, entre la inserción y el aislamiento. Bogotá: Universidad Nacional, Instituto de Estudios Políticos y Editorial Siglo del Hombre.

Pardo, D. y Cardona, D. (1995). "El procedimiento de la certificación y las relaciones entre Colombia y Estados Unidos", Colombia Internacional, 29, 3-6.

Pardo, R. y Tokatlián, J. G. (1988). Política exterior colombiana: ¿De la subordinación a la autonomia? Bogotá: Tercer Mundo Editores / Ediciones Uniandes.

Paoli, L. (2002). The Development of an Illegal Market. Drug Consumption and Trade in Post-Soviet Russia, British Journal of Criminology, 42, 21-39.

Randall, S. (1992), Colombia and the United States: Hegemony and Interdependence. Athens, GA: University of Georgia Press.

(2002), Driven by Drugs: US Policy Towards Colombia. Boulder: Lynne Rienner Pub.

Rouvinski, V. (2014). "Rusia y ALBA: ¿Un matrimonio por conveniencia?” en Bagley y Defort, (Eds.) ¿La hegemonía norteamericana en declive? El desafí del ALBA y la nueva integración latinoamericana del Siglo XXI, Cali: Universidad Icesi, 469-496.

Samper Pizano, E. (1995). Discurso de bienvenida del señor presidente de la República de Colombia, Ernesto Samper Pizano, en la sesión inaugural de la XI Cumbre de Jefes de Estado y de Gobierno del Movimiento de Países No Alineados en Cartagena de Indias, 18 de octubre de 1995, Colombia Internacional, 31, 28-32. 
(1996). Hacia Una Agenda Mundial Contra Las Drogas. Discurso del señor presidente de la República de Colombia, Ernesto Samper Pizano, ante la 51a Asamblea General de las Naciones Unidas 23 de septiembre de 1996, publicado en Samper Pizano, E. (2013). Drogas. Probibición o legalización. Bogotá: Random House Mondadori, 275-283.

(2013a). Drogas. Probibición o legalización. Bogotá: Random

House Mondadori

(2013b). Entrevista realizada el 9 de mayo de 2013 en Cali, Va-

lle. Entrevistador: Vladimir Rouvinski. Archivo privado del entrevistador. Sanchez Cabarcas, F. y Monroy Hernández, C. (2012). "Actores, decisiones y construcción de la política exterior colombiana hacia los Estados Unidos en la era Santos" en Stefan Jhost (Ed.) Colombia: ¿una potencia en desarrollo? Bogotá: Fundación Konrad Adenauer, Colombia, 323-338.

Sierra, A. (1992). Colombianos en Rusia se quedan sin opción. El Tiempo, 10 de febrero.

Stockholm International Peace Research Institute (1998) SIPRI Yearbook 1998. Armaments, Disarmament and International Security. http:// www.sipri.org/yearbook/1998

(1999) SIPRI Yearbook 1999. Armaments, Disarmament and International Security. http://www.sipri.org/yearbook/1999

Stryker, S. and Anne Statham (1985). "Symbolic Interaction and Role Theory." en Gardner Lindzey and Elliot Aronson (Eds.) Handbook of Social Psychology, New York: Random House: 311-378.

Thies C. (2009). Role Theory and Foreign Policy, International Studies Association Compendium Project, Foreign Policy Analysis section, http://myweb. uiowa.edu/bhlai/workshop/role.pdf

(2012). International Socialization Processes v. Israeli National Role Conceptions: Can Role Theory Integrate IR Theory and Foreign Policy Analysis? Foreign Policy Analysis 8, 25-46 (2014), Role Theory and Foreign Policy Analysis in Latin America. Foreign Policy Analysis, 0, 1-20 (DOI: 10.1111/fpa.12072)

Tickner, A. (2000). Tensiones y Contradicciones en los Objetivos de la Política Exterior Estadounidense en Colombia. Colombia Internacional, (49-50), 39-61. 
(2007). Intervención por invitación: Claves de la política exterior colombiana y de sus debilidades principales. Colombia Internacional, 65, 90-111. (2008). Latin America IR and the Primacy of Lo Práctico. International Studies Review, 10, 735-748.

(2014). Legalización de las drogas: entre las políticas de seguridad y la salud pública. Trans-Pasando Fronteras, (5), 21-28.

Tokatlian, J. (1997). De Rusia con Amor. El Tiempo, 23 de noviembre (2008). La Construcción de un "Estado Fallido" en la Politica Mundial: el Caso de las Relaciones Entre Estados Unidos y Colombia. Análisis Politico, 64, 67-104

Trenin, D. (2007). Russia Redefines Itself and its Relations with the West. The Washington Quarterly, 30 (2), 95-96.

Trushin, Alexander. (1998). Mi-17 en el servicio de América Latina [en Ruso]. Aviapanorama, No.5, s/p.

United States Congress (1996). House. Committee on Government Reform. Subcommittee on Criminal Justice, Drug Policy, and Human Resources. Overall U.S. Counter Narcotics Policy Towards Colombia. 11 September 1996. Washington, DC.: U.S. Government Printing Office

Velosa Porras, E. (2012a). Las ideas en la construcción de las relaciones internacionales. Papel Politico, 17: 655-677.

(2012b). "Las ideas y la política exterior colombiana: una mirada desde la teoría del rol nacional y el institucionalismo discursivo" en Stefan Jhost (Ed.) Colombia: ¿una potencia en desarrollo? Bogotá: Fundación Konrad Adenauer, Colombia, 39-54.

Walker, S (1981). The Correspondence between Foreign Policy Rhetoric and Behavior: Insights from Role Theory and Exchange Theory. Behavioral Science 26: 272-281.

(1987). "Role Theory and Foreign Policy Analysis: An Evaluation" en Stephen Walker, (ed.), Role Theory and Foreign Policy Analysis, Durham, NC: Duke University Press, 241-259 
